\title{
Propriedades da resina composta bulk fill: uma revisão da literatura
}

\author{
Bullk fill resin composite properties: a literature review \\ Propriedades de la resina compuesta bulk fill: revisíon de la literatura
}

Recebido: 21/09/2021 | Revisado: 03/10/2021 | Aceito: 05/10/2021 | Publicado: 09/10/2021

\author{
Bianca Berto Rodrigues \\ ORCID: https://orcid.org/0000-0002-4164-8677 \\ Universidade Estadual da Paraíba, Brasil \\ E-mail: bianca.berto.9@gmail.com \\ Lilian Juliana Torres Silva \\ ORCID: https://orcid.org/0000-0002-6753-6219 \\ Universidade Estadual da Paraíba, Brasil \\ E-mail: lilianjuliana8@gmail.com \\ Geovanna Caroline Brito da Silva \\ ORCID: https://orcid.org/0000-0002-8943-5638 \\ Universidade Estadual da Paraíba, Brasil \\ E-mail: geovannacarolineb@gmail.com \\ Henrique Souto Vieira \\ ORCID: https://orcid.org/0000-0003-3672-9437 \\ Universidade Estadual da Paraíba, Brasil \\ E-mail: hsouto15@gmail.com \\ Fernanda Campos \\ ORCID: https://orcid.org/0000-0002-0100-2740 \\ Universidade Estadual da Paraíba, Brasil \\ E-mail: fernandacampos@gmail.com \\ Rodrigo Barros Esteves Lins \\ ORCID: https://orcid.org/0000-0002-8224-6578 \\ Universidade Estadual da Paraíba, Brasil \\ E-mail: lins@servidor.uepb.edu.br
}

\begin{abstract}
Resumo
Atualmente, a resina composta tornou-se a primeira opção de escolha para os tratamentos restauradores, pois apresenta grandes benefícios como uma maior reprodução de detalhes, mimetizando a estrutura dentária e possibilitando procedimentos minimamente invasivos e seguros. Entretanto, os problemas resultantes da contração de polimerização ainda constituem um desafio no que tange a longevidade e o sucesso clínico da restauração, sendo contornada através da técnica incremental. Como alternativa e com o propósito de otimizar o tempo clínico em restaurações posteriores, surgiu no mercado as resinas compostas de baixa contração de polimerização que são denominadas resinas bulk fill, que possibilitam a sua utilização na forma incrementos únicos com espessuras superiores a $4 \mathrm{~mm}$. Esse material vem sendo amplamente difundido na clínica odontológica, apresentando benefícios como menor tempo clínico, maior profundidade de polimerização, menor contração e menor tensão de polimerização geradas às paredes da cavidade. As diferenças das propriedades e na composição dessa resina acontecem conforme as variações definidas pelo fabricante. Assim sendo, é imprescindível o conhecimento da composição química do material a ser utilizado na clínica, adequando-se às indicações postas pelo fabricante, a fim de que se possa alcançar resultados satisfatórios e duradouros. Dessa forma, este artigo teve como objetivo revisar as principais características e propriedades da resina composta bulk fill, bem como suas indicações, vantagens e desvantagens.
\end{abstract}

Palavras-chave: Resinas compostas; Materiais dentários; Polimerização.

\begin{abstract}
Currently, resin composite has become the first choice for restorative treatments, as it has great benefits such as greater reproduction of details, mimicking the tooth structure and enabling minimally invasive and safe procedures. However, the problems resulting from polymerization shrinkage stress are still a challenge regarding the longevity and clinical success of the restoration, being circumvented through the incremental technique. As an alternative and with the purpose of optimizing the clinical time in posterior restorations, resin composites with low shrinkage stress polymerization appeared on the market, which are named bulk fill resins, which enable their use in the form of unique increments with thicknesses greater than $4 \mathrm{~mm}$. This material has been widely used in the dental clinic, presenting benefits such as shorter clinical time, greater depth of polymerization, less polymerization shrinkage stress and less polymerization stress generated to the cavity walls. The differences in the properties and composition of this resin occur according to the variations defined by the manufacturer. Therefore, it is essential to know the chemical composition of the material to be used in the clinic, adapting to the indications given by the manufacturer, in order to
\end{abstract}


achieve satisfactory and lasting results. Thus, this article aimed to review the main characteristics and properties of bulk fill composite resin, as well as its indications, advantages and disadvantages.

Keywords: Composite resins; Dental materials; Polymerization.

\section{Resumen}

Actualmente, la resina compuesta se ha convertido en la primera opción para los tratamientos restauradores, ya que tiene grandes beneficios como una mayor reproducción de los detalles, mimetizando la estructura del diente y permitiendo procedimientos mínimamente invasivos y seguros. Sin embargo, los problemas resultantes de la contracción de la polimerización siguen siendo un desafío en cuanto a la longevidad y el éxito clínico de la restauración, y se eluden mediante la técnica incremental. Como alternativa y con el propósito de optimizar el tiempo clínico en las restauraciones posteriores, aparecieron en el mercado resinas compuestas con baja contracción de polimerización, que se denominan resinas bulk fill, que permiten su uso en forma de incrementos únicos con espesores superiores a $4 \mathrm{~mm}$. Este material ha sido ampliamente difundido en la clínica dental, presentando beneficios como menor tiempo clínico, mayor profundidad de polimerización, menor contracción de polimerización y menor estrés de polimerización generado en las paredes de la cavidad. Las diferencias en las propiedades y composición de esta resina se producen según las variaciones definidas por el fabricante. Por ello, es fundamental conocer la composición química del material a utilizar en la clínica, adecuándose a las indicaciones dadas por el fabricante, con el fin de conseguir resultados satisfactorios y duraderos. Así, este artículo tuvo como objetivo revisar las principales características y propiedades de la resina compuesta bulk fill, así como sus indicaciones, ventajas y desventajas.

Palabras clave: Resinas compuestas; Materiales dentales; Polimerización.

\section{Introdução}

Diante da constante busca por materiais restauradores com características mecânicas e estéticas similares às estruturas dentais, as resinas compostas surgem para suprir essa demanda clínica (Chesterman et al., 2017). Entretanto, as resinas compostas convencionais apresentam algumas limitações, como a inserção limitada de incremento, evitando-se a união de paredes opostas da cavidade; e as consequências decorrentes da contração e tensão de polimerização, como: a desadaptação marginal, microinfiltração, surgimento de cárie secundária, sensibilidade pós-operatória e até trincas no esmalte e fratura de cúspide, os quais podem levar ao insucesso do procedimento restaurador (Lins et al., 2019).

Além disso, o fator de configuração cavitária (fator C), razão entre a área de superfície livre e a área da superfície aderida da resina composta em relação às paredes da cavidade, pode propiciar falhas na restauração, pois quanto maior for o fator C, maior será a tensão de contração gerada na interface de união (Fu; Aregawi; Fok, 2020). Como forma de contornar estas limitações, indica-se a inserção da resina composta em pequenos incrementos, com espessura de até $2 \mathrm{~mm}$ a fim de controlar a contração do material resinoso (Ferracane \& Hilton, 2016).

Assim exposto, uma forma de lidar com dificuldades, como a tensão de polimerização e frente a necessidade do aprimoramento tanto das técnicas restauradoras, quanto dos materiais restauradores, surgiu no mercado o desenvolvimento de uma resina composta de baixa contração de polimerização que permite ser utilizada como preenchimento em incremento único, denominada de resina composta Bulk Fill (Zorzin et al., 2015). Esse material possibilita a inserção de um incremento de maior espessura, superiores a $4 \mathrm{~mm}$, facilitando o procedimento restaurador e otimizando o tempo clínico em cavidades profundas, largas e de grande comprometimento coronário (Zorzin et al., 2015).

Os fabricantes realizaram diversas alterações na composição da resina composta a fim de proporcionar a utilização de maiores volumes de material, que serão fotopolimerizados por um menor tempo. Tais alterações estão relacionadas à matriz monomérica, à quantidade, dimensão e tratamento de superfície das partículas de carga, e à utilização de sistemas fotoiniciadores alternativos (Bayraktar et al., 2017; Bucuta \& Ilie; 2000; Gan et al., 2018; Ilie; Bucuta \& Draenert, 2013).

Destaca-se que, devido a utilização de sistemas fotoiniciadores alternativos, diferindo dos tradicionais à base de canforoquinona, exige-se a compatibilidade destes sistemas à fonte de luz polimerizadora disponível, devendo a faixa espectral ser compatível com fotoiniciadores presentes nas resinas compostas bulk fill (Gan et al., 2018). Além disso, o ganho nas características ópticas à resina composta é significativo, pois os referidos fotoiniciadores alternativos não apresentam a 
coloração amarelada da canforoquinona, facilitando a versatilidade da escala de cores disponível pelo fabricante (Gan et al., 2018).

Similarmente à resina composta convencional, a resina composta bulk fill apresenta-se em diferentes viscosidades em relação a composição da matriz orgânica e inorgânica, sendo de alta viscosidade, também denominada de restauradora ou esculpível; e de baixa viscosidade, também denominada de fluida ou flow, ou ainda flowable (Sebold et al., 2020).

A resina composta bulk fill pode ser empregada em diferentes técnicas restauradoras, como: a técnica de dois passos, onde utiliza-se a resina composta bulk fill de baixa viscosidade como base e uma resina composta convencional como cobertura oclusal; a técnica de dois passos adaptada, a qual utiliza-se uma resina composta bulk fill de baixa viscosidade como base e uma resina composta bulk fill de alta viscosidade como cobertura oclusal; e a técnica de passo único, a qual trabalha-se apenas com a resina composta bulk fill de alta viscosidade em incremento único (Hirata et al., 2015; Sebold et al., 2020). Além disso, uma segunda geração de resinas compostas bulk fill utiliza-se da inserção da resina composta na cavidade através de vibração sônica, produzindo uma significativa redução na viscosidade do material, o qual permite melhor inserção e adaptação da resina composta nas paredes da cavidade, e ao cessar a ativação sônica, gradativamente o material torna-se menos viscoso, facilitando a etapa de escultura oclusal da restauração (Monterubbianesi et al., 2016).

Todavia, apesar das vantagens e resultados promissores destes materiais presentes em estudos científicos, estes são relativamente recentes no mercardo, possuindo uma composição e características de manuseio diversas e marca-dependente. Além disso, apresentam propriedades físicas e mecânicas diferentes, e portanto, exigindo-se a realização de estudos futuros sobre esse material (Czaschc \& Ilie, 2013). Deste modo, esta revisão da literatura tem como objetivo atualizar o leitor em relação a atual circunstância da resina composta bulk fill com base em estudos científicos, relacionando às características mecânicas e ópticas deste material.

\section{Metodologia}

Este estudo caracterizou-se por uma busca bibliográfica nas bases de dados eletrônicos PubMed e BVS (Biblioteca Virtual em Saúde), aplicando-se termos MeSh e Descritores em Ciências da Saúde, respectivamente. Os termos pesquisados foram: "composite resins", "resina composta", "resin composite bulk fill", "resina composta bulk fill" e "resina bulk fill propriedades" associados através de operadores booleanos "AND" e/ou "OR". Outra estratégia utilizada foi a busca manual em listas de referências dos artigos identificados/selecionados.

Como critérios de inclusão, foram adotados os artigos escritos em inglês, espanhol e português, os quais se enquadravam no enfoque deste trabalho de revisão, possuindo alto impacto e de grande relevância para a literatura científica. Além disso, foram incluídos estudos que apresentavam disponibilidade do texto na íntegra e clareza no detalhamento metodológico utilizado. O tipo de estudo não foi fator limitador para a seleção dos estudos, podendo ser estudos in vitro, in vivo, em animais, revisões da literatura, de escopo ou sistemática, e estudos clínicos randomizados. Caso o artigo não estivesse disponível, foram enviadas solicitações via e-mail para os autores correspondentes para a disponibilidade dos mesmos na íntegra. Foram excluídos os artigos que não apresentaram relevância clínica sobre o tema abordado.

Os artigos buscados nas plataformas científicas foram importados para programa eletrônico Mendeley (Mendeley software, Elsevier, Londres, GB) utilizado para a seleção e leitura dos artigos, incialmente pelo título e resumo, e posteriormente pelo texto completo.

\section{Discussão}

Com os crescentes avanços da Odontologia para oferecer tratamentos minimamente invasivos, a resina composta tornou-se a primeira opção para as restaurações diretas (Rodrigues et al., 2021). Maior estética e propriedades mecânicas 
aprimoradas quando comparada a outros materiais restauradores foram fatores que corroboraram com a sua aceitação, além de reestabelecer a forma anatômica e a função dos dentes acometidos por uma lesão de cárie ou ocorrência de fraturas dentais (Demarco et al., 2012; Laegreid et al., 2014; Rodrigues et al., 2021). Entretanto, apesar de seu preparo conservador em relação às técnicas de preparo cavitário para o amálgama, a ocorrência de contração advinda do processo de polimerização ainda é considerada um grande desafio, podendo interferir na longevidade e sucesso clínico do procedimento restaurador (Bicalho et al., 2014; Fleming et al., 2005; Kleverlaan \& Feilzer, 2005; Lins et al., 2019a).

Sendo assim, como forma de contornar essas limitações relacionado ao estresse gerado pela contração de polimerização e evitar desafios relacionados à profundidade de polimerização, a técnica incremental é a alternativa mais usada na rotina clínica. Porém, existem algumas desvantagens nessa técnica, como espaços vazios que podem ficar aprisionados entre essas camadas, risco de contaminação entre as camadas, dificuldade na colocação dos pequenos incrementos em cavidades de difícil acesso e aumento do tempo clínico na cadeira odontológica (Bicalho et al., 2014; Abbas et al., 2003).

\section{Desenvolvimento e benefícios}

Dessa forma, com o decorrer dos anos, os compósitos bulk fill, de preenchimento único têm ganhado grande destaque para as restaurações diretas posteriores pois possuem vantagens como: a inserção de um único incremento ou incrementos de maiores espessuras, em um baixo tempo de fotoativação, apresentando boas propriedades estéticas, capacidade de proteção aos tecidos dentais durante o preparo, além de possuir um maior custo-benefício quando comparado aos materiais restauradores indiretos (Türkün, Aktener, Ateş, 2003).

As resinas compostas bulk fill permitem a inserção do material em incrementos superiores a $4 \mathrm{~mm}$ de espessura, sendo indicado um baixo tempo de fotoativação por parte dos fabricantes (Núñez et al., 2015). Isso ocorre pelo aumento da translucidez dessas resinas compostas, tornando mais facilitada a passagem da luz do fotopolimerizador às camadas mais profundas do incremento inserido (El-Safty et al., 2012). Além disso, essas resinas podem ser clinicamente inseridas por meio de 3 técnicas de restauração: a técnica de bulk de duas etapas (usando bulk-fill fluida coberto com material de resina composta convencional), a técnica de bulk com ativação sônica (usando bulk-fill fluida com ativação sônica) e a técnica em massa (usando tipo pasta ou preenchimento em massa regular) (Núñez et al., 2015).

Como relatado anteriormente, as resinas compostas bulk fill são compósitos resinosos que sofreram algum tipo de alteração em sua composição química, a fim de melhorar as suas propriedades mecânicas e ópticas, e favorecer a técnica restauradora, sendo estas alterações relacionadas com modificação na matriz orgânica, por meio da adição e mistura de diferentes monômeros resinosos; adição de partículas de carga com tamanhos variados, com tratamentos de superfície, favorecendo no alcance da luz do fotopolimerizador a camadas mais profundas devido a alterações no índice de refração da luz; e ainda, adição de sistemas fotoativadores alternativos, como o Ivocerin ${ }^{\circledR}$ (derivado de dibenzoil germânico) (Ivoclar Vivadent) e TPO (óxido de mono-alquil fosfina), os quais possuem diferentes picos de comprimento de onda na faixa do espectro da radiação, sendo necessária a utilização de fontes de luz que apresentem um alcance do comprimento de onda compatível com os sistemas fotoiniciadores presentes nestes materiais (Boaro et al., 2013; Fronza et al., 2017).

Estudos laboratoriais que apresentam como objetivo caracterizar estes materiais apresentam grande relevância científica, visto que estes materiais serão utilizados clinicamente em seres humanos. Dentre os critérios de caracterização, temos a dureza superficial (medida como microdureza, por meio de testes de Vickers ou Knoop), definida como a resistência ao recorte permanente ou penetração com o tempo, deve ser criteriosamente avaliada, pois estas quando em função, estarão expostas a grandes áreas de força mastigatória (Galvão et al., 2013; Tarle et al., 2015; Hoshino et al., 2021). Além disso, em um estudo desenvolvido por Flury e colaboradores (2014) demonstrou que resinas compostas bulk fill, diante da aplicação de incrementos mais espessos não reduziram a resistência de união, diferentemente das resinas convencionais, as quais 
diminuíram a resistência de união em relação ao aumento da espessura dos incrementos (Simon Flury, Anne Peutzfeldt \& Adrian Lussi, 2014).

\section{Contração de polimerização}

A reação de polimerização de materiais resinosos ocorre pela quebra das duplas ligações de carbono, transformando moléculas monoméricas em redes poliméricas. Em pesquisas laboratoriais, essa transformação é mensurada pelo grau de conversão monomérico no material. Tal reação produz consequências à restauração, relacionadas à contração volumétrica e linear do material e à tensão produzida na interface adesiva frente ao processo de polimerização (Leprince et al., 2013; Lins et al., 2019). Esta contração de polimerização está diretamente relacionada com o tipo de resina composta utilizada, com a quantidade de material inserido na cavidade, com o tamanho e a natureza dos monômeros, com o módulo de elasticidade, o tipo e a técnica de fotopolimerização e pelo fator de configuração cavitário (fator C) (Yoshikawa et al., 1999; Ferracane \& Hilton, 2016; Rueggeberg et al., 2017).

Em um estudo avaliando os efeitos da espessura da resina composta na microdureza e nas propriedades ópticas de compósitos de resina bulk-fill desenvolvido por Kim e colaboradores, destaca-se que a primeira classe de resinas compostas bulk fill criada apresentava-se com uma consistência fluida, e que a estratégia utilizada para conseguir uma característica de preenchimento único apresentando baixa contração de polimerização foi possível pelo aumento da translucidez do material resinoso e adição de sistemas fotoiniciadores alternativos (Kim et al., 2015).

Em um estudo clínico e laboratorial realizado por Boaro e colaboradores (2019) cujo objetivo foi analisar a contração de polimerização de uma resina composta bulk fill com resinas compostas convencionais, foi observado que resinas compostas bulk fill com viscosidade regular possuíam contração de polimerização similar às convencionais, à medida que, compósitos bulk fill de viscosidade fluida demonstravam menor contração de polimerização em relação aos compósitos fluidos convencionais, sendo assim, foi possível concluir que a contração de polimerização de ambas as resinas compostas eram dependentes da sua viscosidade, que os compósitos bulk fill apresentaram desempenho semelhante ou melhor em relação às resinas compostas convencionais sobre a tensão de polimerização, deflexão da cúspide, desadaptação marginal, grau de conversão, resistência flexural, e resistência à fratura (Boaro et al., 2019).

Estudos laboratoriais que avaliaram a contração linear de polimerização e a tensão gerada pelo processo de polimerização de resinas compostas convencionais e bulk fill por meio de fibra óptica observaram que resinas compostas bulk fill apresentam comportamento similar às resinas compostas convencionais, contudo, induzem menor tensão de polimerização na interface adesiva, e que este comportamento pode estar relacionado com o menor estresse proporcionado pelas múltiplas camadas de incrementos indicados pela técnica convencional, o qual induz vetores de tensão em diferentes direções à medida em que são inseridos diversos incrementos em uma mesma cavidade; e além disso, de fato os compósitos bulk fill apresentam menor contração de polimerização a depender do fabricante, como por exemplo, resinas compostas bulk fill à base de uma rede de copolímeros orgânico-inorgânico mais denso que os monômeros convencionais de dimetacrilato, denominados de Ormocer (Lins et al 2019a; Lins et al., 2019b).

\section{Adaptação marginal}

A adaptação marginal do material restaurador depende de diversos fatores, como a habilidade do profissional em adaptar corretamente a resina composta às paredes da cavidade com espátulas apropriadas; a quantidade de material inserida na cavidade e a sua característica de contração de polimerização; em relação a técnica adesiva de escolha; e também o tipo de substrato em que se realiza a adesão, seja em esmalte ou dentina, além da sua integridade estrutural e química (Geerts, Bolette, Seidel \& Gueders, 2012; Gregor, Bortolotto, Feilzer \& Krejci, 2013). 
A inserção da resina composta bulk fill em cavidades profundas deve ser realizada com cautela, visto que se trata de um incremento de grande espessura em uma situação clínica desafiadora, podendo comprometer a adaptação marginal nas paredes internas da cavidade pelo aprisionamento de bolhas ou formação de gaps marginais. No estudo de Alqudaihi et al. (2019) realizaram uma comparação in vitro sobre a adaptação interna de diferentes materiais resinosos, os quais puderam concluir que a técnica restauradora incremental desempenha melhor adaptação interna na cavidade em relação à técnica restauradora bulk fill, com incremento único, e que cada tipo de resina composta bulk fill apresenta um comportamento diferente. Além disso, a degradação marginal por meio de termociclagem induziu a ocorrência de desadaptação marginal, mas não influenciou na adaptação interna da cavidade.

Resina composta bulk fill de ativação sônica é defendido cientificamente como um material que induz melhor adaptação marginal devido a sua facilidade em se adaptar às paredes da cavidade pela capacidade de variar a viscosidade pela vibração sônica, além de ser utilizado também em incrementos espessos, em torno de $5 \mathrm{~mm}$, e fotopolimerizado por curto período de tempo com aparelhos de alta potência (Gamarra, Borges, Júnior \& Spohr, 2017). Contudo, esta técnica necessita da utilização de aparelhos mais caros, que induzam a vibração sônica e que são compatíveis apenas com os produtos do fabricante, portanto, caracterizando uma limitação da sua utilização clínica.

\section{Resina composta bulk fill na odontopediatria}

Outra aplicação clínica para a resina composta bulk fill é a utilização em dentes decíduos, dentre outras vantagens, o fato de ser em incremento único, o que diminui o tempo de atendimento clinico e permite maior rapidez por parte do profissional em realizar o procedimento clínico em pacientes que não sejam colaboradores (Mosharrafian, Shafizadeh \& Sharifi, 2019). Eventos adversos em pacientes jovens são caracterizados pela presença de amplas lesões cariosas ou fratura dental, os quais comprometem a estrutura coronário do dente decíduo de forma significativa, sendo assim, a aplicação da resina composta bulk fill nesse cenário clínico apresenta-se bem indicado, comprovadamente avaliado desde estudos laboratoriais à estudos clínicos randomizados (Akman \& Tosun, 2020; Ehlers et al., 2019; Mosharrafian, Shafizadeh \& Sharifi, 2019).

Dois estudos clínicos randomizados desenvolvido em 30 e 32 crianças, respectivamente, atuando em 160 e 100 restaurações classe II, comparou diferentes tipos de resinas compostas bulk fill (Sonicfill, X-tra fil e Venus bulk fill) e outros materiais restauradores, como o cimento ionômero de vidro, com objetivo de observar como essa esses materiais se comportavam no decorrer de 1 ano de acompanhamento clínico, e pôde-se observar que a resina composta bulk fill superou o cimento de ionômero de vidro em relação à adaptação marginal e retenção na cavidade, demonstrando um ótimo desempenho clinico dessas resinas compostas, assim como a resina composta bulk fill de baixa viscosidade também demonstrou ser um material alternativo para restaurações em pacientes pediátricos (Akman \& Tosun, 2020; Ehlers, Gran, Callaway, Azrak, \& Ernst, 2019).

\section{Estudos clínicos e revisões sistemáticas}

Diversos estudos clínicos presentes na literatura científica demonstram certa similaridade aos estudos laboratoriais, ou seja, apresentam excelentes resultados clínicos com base em parâmetros relacionados à retenção do material na cavidade, nas características estéticas, no grau de degradação do material tanto no corpo da restauração, como na interface adesiva, assim como eventos adversos, como sensibilidade, ocorrência de trincas ou fraturas, e ainda o desenvolvimento de lesão de cárie secundária. Dentre esses, pode-se observar que ambos os tipos de resina composta (convencional ou bulk fill) possuem comportamento biomecânico bastante similar, ou mais especificamente, o desempenho clínico é material dependente, seja ele qual o tipo de resina composta seja. Para tanto, estudos longitudinais em diferentes situações clínicas, como restaurações em dentes posteriores classe I (Al-Sheikh, 2019), classe II (Alkurdi \& Abboud, 2016; Balkaya \& Arslan, 2020; Balkaya; Arslan \& 
Pala, 2019; Bayraktar et al., 2017; Guney \& Yazici, 2019; Yazici et al., 2017), ou ambas as classes (Arhun; Celik; Yamanel, 2010; Berti et al., 2020; Durão et al., 2020; Heck et al., 2019; Manhart; Chen \& Hickel, 2010) e em lesões cervicais não cariosas (LCNC) (Canali et al., 2019; Correia et al., 2020; Vildósola et al., 2019) corroboram estes achados, assim como, revisões sistemáticas com meta-análises refutam estes achados e conclusões (Arbildo-Veja et al., 2020; Boaro et al., 2019; Kruly et al., 2018; Veloso et al., 2019).

\section{Conclusão}

Diante do exposto, conclui-se que o uso de resina composta bulk fill vem sendo amplamente difundido na prática clínica devido às diversas vantagens desse material, como o menor tempo clínico, uma maior profundidade de polimerização, na qual possibilita fotopolimerizar camadas mais profundas do incremento decorrente da sua maior translucidez, bem como menor contração de polimerização e menor tensão de polimerização geradas às paredes da cavidade, diminuindo portanto, a susceptibilidade de eventos adverosos relacionados às limitações dos materiais resinosos e garantindo maior longevidade clínica à restauração. As diferenças das propriedades e na composição dessa resina acontecem conforme as variações definidas pelo fabricante. Assim sendo, é imprescindível o conhecimento da composição química do material a ser utilizado na clínica, adequando-se às indicações postas pelo fabricante, a fim de que se possa alcançar resultados satisfatórios e duradouros.

\section{Referências}

3M ESPE. Filtek Bulk Fill Posterior Restorative. Technical Product Profile. http://multimedia.3m.com/mws/media/976634O/ filtek-bulk-fill-posteriorrestorative-technical-product-profile.pdf. Accessed September 2017.

Abbas, G., Fleming, G. J., Harrington, E., Shortall, A. C., \& Burke, F. J. (2003). Cuspal movement and microleakage in premolar teeth restored with a packable composite cured in bulk or in increments. $J$ Dent, 31(6), 437-444.

Agência do Clima e Poluição. Revisão das experiências norueguesas com a eliminação progressiva do uso de amálgama dentário. TA 2946, Oslo, Noruega 2012 .

Akman, H., \& Tosun, G. (2020). Clinical evaluation of bulk-fill resins and glass ionomer restorative materials: A 1-year follow-up randomized clinical trial in children. Niger J Clin Pract, 23(4), 489-497. https://doi.org/10.4103/njcp.njcp_519_19.

Al-Sheikh, R. (2019). Effects of Different Application Techniques on Nanohybrid Composite Restorations Clinical Success. The Open Dentistry Journal, 13, 228-235. https://doi.org/10.2174/1874210601913010228.

Alkurdi, R. M., \& Abboud, S. A. (2016). Clinical evaluation of class II composite: Resin restorations placed by two different bulk-fill techniques. J Orofac Sci, 8, 34-9. https://doi.org/10.4103/0975-8844.181926.

Alqudaihi, F. S., Cook, N. B., Diefenderfer, K. E., Bottino, M. C., \& Platt, J. A. (2019). Comparison of Internal Adaptation of Bulk-fill and Increment-fill Resin Composite Materials. Oper Dent, 44(1), E32-E44. https://doi.org/10.2341/17-269-L.

Arbildo-Vega, H. I., Lapinska, B., Panda, S., Lamas-Lara, C., Khan, A. S., \& Lukomska-Szymanska, M. (2020). Clinical Effectiveness of Bulk-Fill and Conventional Resin Composite Restorations: Systematic Review and Meta-Analysis. Polymers (Basel), 12, 1786. https://doi.org/10.3390/polym12081786.

Arhun, N., Celik, C., \& Yamanel, K. (2010). Clinical evaluation of resin-based composites in posterior restorations: two-year results. Oper Dent, 35, 397-404. https://doi.org/10.2341/09-345-C.

Balkaya, H., \& Arslan, S. (2020). A Two-year Clinical Comparison of Three Different Restorative Materials in Class II Cavities. Oper Dent, 45, e32-e42. https://doi.org/10.2341/19-078-C.

Balkaya, H., Arslan, S., \& Pala, K. (2019). A randomized, prospective clinical study evaluating effectiveness of a bulk-fill composite resin, a conventional composite resin and a reinforced glass ionomer in Class II cavities: one-year results. J Appl Oral Sci, 7, e20180678. http://dx.doi.org/10.1590/1678-77572018-0678.

Baur, V., \& Ilie, N. (2013). Repair of dental resin-based composites. Clin Oral Investig, 17(2), 601-608.

Bayraktar, Y., Ercan, E., Hamidi, M. M., \& Çolak, H. (2017). One-year clinical evaluation of different types of bulk-fill composites. J Investig Clin Dent, 8(2). https://doi.org/10.1111/jicd.12210.

Berti, L. S., Turssi, C. P., Amaral, F. L., Basting, R. T., Junqueira, J. L. C., Panzarella, F. K., Reis, A. F., \& França, F. M. (2020). Clinical and radiographic evaluation of high viscosity bulk-fill resin composite restorations. Am J Dent, 33, 213-217.

Benetti, A. R., Havndrup-Pedersen, C., Honoré, D., Pedersen, M. K., \& Pallesen, U. (2015). Bulk-fill resin composites: polymerization contraction, depth of cure, and gap formation. Oper Dent, 40(2), 190-200. 
Bicalho, A. A., Pereira, R. D., Zanatta, R. F., Franco, S. D., Tantbirojn, D., Versluis, A., \& Soares, C. J. (2014). Incremental filling technique and composite material--part I: cuspal deformation, bond strength, and physical properties. Oper Dent, 39(2), 71-82.

Boaro, L. C. C., Lopes, D. P., de Souza, A. S. C., Nakano, E. L., Perez, M. D. A., Pfeifer, C. S., \& Gonçalves, F. (2019). Clinical performance and chemicalphysical properties of bulk fill composites resin - a systematic review and meta-analysis. Dent Mater, 35, e249-e264. https://doi.org/10.1016/j.dental.2019.07.007.

Bucuta, S., \& Ilie, N. (2014). Light transmittance and micro-mechanical properties of bulk fill vs. conventional resin based composites. Clin Oral Investig, $18(8), 1991-2000$

Canali, G. D., Ignácio, S. A., Rached, R. N., \& Souza, E. M. (2019). One-year clinical evaluation of bulk-fill flowable vs. regular nanofilled composite in noncarious cervical lesions. Clin Oral Investig, 23, 889-897. https://doi.org/10.1007/s00784-018-2509-8.

Chesterman, J., Jowett, A., Gallacher, A., \& Nixon, P. (2017). Bulk-fill resin-based composite restorative materials: a review. Br Dent J, 222(5), 337-344.

Corral Núñez C., Vildósola Grez P., Bersezio Miranda C., Alves Dos Campos E., Fernández Godoy E. (2015) Estado da arte dos compósitos à base de resina de enchimento a granel: Uma revisão. Rev. Fac. Odontol, 27: 177-196. https://doi.org/10.17533

Correia, A., Jurema, A., Andrade, M. R., Borges, A., Bresciani, E., \& Caneppele, T. (2020). Clinical Evaluation of Noncarious Cervical Lesions of Different Extensions Restored With Bulk-fill or Conventional Resin Composite: Preliminary Results of a Randomized Clinical Trial. Oper Dent, 45, e11-e20. https://doi.org/10.2341/18-256-C.

Czasch, P., \& Ilie, N. (2013). In vitro comparison of mechanical properties and degree of cure of bulk fill composites. Clin Oral Investig, 17(1), $227-235$.

Demarco, F. F., Corrêa, M. B., Cenci, M. S., Moraes, R. R., \& Opdam, N. J. (2012). Longevity of posterior composite restorations: not only a matter of materials. Dent Mater, 28(1), 87-101

Dentsply. Surefil SDR Bulk Fill Flowable. Technical Product $\quad$ Profile. Disponível em: http://www.dentaltown.com/Images/Dentaltown/magimages/0216/http://www.dentaltown.com/Images/Dentaltown/magimages/0216/

Durão, M. A., Andrade, A. K. M., Santos, M. D. C. M. D. S., Montes, M. A. J. R., \& Monteiro, G. Q. M. (2020). Clinical Performance of Bulk-Fill Resin Composite Restorations Using the United States Public Health Service and Federation Dentaire Internationale Criteria: A 12-Month Randomized Clinical Trial. Eur J Dent, 26. https://doi.org/10.1055/s-0040-1718639.

Ehlers, V., Gran, K., Callaway, A., Azrak, B., \& Ernst, C. P. (2019). One-year Clinical Performance of Flowable Bulk-fill Composite vs Conventional Compomer Restorations in Primary Molars. J Adhes Dent, 21(3), 247-254. https://doi.org/10.3290/j.jad.a42519.

El-Safty S, Akhtar R, Silikas N, Watts DC. Nanomechanical properties of dental resin-composites. (2012). Dent Mater. Dec;28(12):1292-300. https://doi.org/10.1016/j.dental.2012.09.007.

Ferracane, J. L., \& Hilton, T. J. (2016). Polymerization stress--is it clinically meaningful?. Dent Mater, 32, 1-10. https://doi.org/10.1016/j.dental.2015.06.020.

Fu J, Aregawi WA, Fok ASL. (2020) Mechanical manifestation of the C-factor in relation to photopolymerization of dental resin composites. Dent Mater, 36(8), 1108-1114. https://doi.org//10.1016/j.dental.2020.05.004.

Fleming, G. J., Hall, D. P., Shortall, A. C., \& Burke, F. J. (2005). Cuspal movement and microleakage in premolar teeth restored with posterior filling materials of varying reported volumetric shrinkage values. $J$ Dent, 33(2), 139-146.

Flury, S., Peutzfeldt, A. \& Lussi, A. (2014). Influence of increment thickness on microhardness and dentin bond strength of bulk fill resin composites. Dent Mater, 30(10), 1104-1112. https://doi: 10.1016/j.dental.2014.07.001.

Fronza, B. M., Ayres, A., Pacheco, R. R., Rueggeberg, F. A., Dias, C., \& Giannini, M. (2017). Characterization of Inorganic Filler Content, Mechanical Properties, and Light Transmission of Bulk-fill Resin Composites. Oper Dent, 42(4), 445-455. https://doi.org/10.2341/16-024-L.

Galvão, M. R., Caldas, S. G., Bagnato, V. S., de Souza Rastelli, A. N., de Andrade, M. F. (2013). Evaluation of degree of conversion and hardness of dental composites photo-activated with different light guide tips. Eur J Dent. 7(1), 86-93.

Gamarra, V. S. S., Borges, G. A., Júnior, L. H. B., \& Spohr, A. M. (2018). Marginal adaptation and microleakage of a bulk-fill composite resin photopolymerized with different techniques. Odontology, 106(1), 56-63. doi: 10.1007/s10266-017-0294-5.9 Jan-Feb;16(1):69-77. https://doi.org/10.18502/fid.v16i1.1112.

Gan, J. K., Yap, A. U., Cheong, J. W., Arista, N., \& Tan, C. (2018). Bulk-Fill Composites: Effectiveness of Cure With Poly- and Monowave Curing Lights and Modes. Oper Dent, 43, 136-143. https://doi.org/10.2341/16-304-L.

Geerts, S., Bolette, A., Seidel, L., \& Gueders, A. (2012). An in vitro evaluation of leakage of two etch and rinse and two self-etch adhesives after thermocycling. Int J Dent, 12, 1-7.

Gregor, L., Bortolotto, T., Feilzer, A. J., \& Krejci, I. (2013). Shrinkage kenetics of a methacrylate- and a silorane-based resin composite: effect on marginal integrity. J Adhes Dent, 15, 245-50.

Grigoletto, J. C., Oliveira, A. S., Muñoz, S. I. S., Alberguini, L. B. A., \& Takayanagui, A. M. M. (2008). Occupational risk due to use of mercury in dentistry: a bibliographic review. Ciências saúde coletiva, 13(2), 533-542. https://doi.org/10.1590/S1413-81232008000200029.

Guney, T., \& Yazici, A. R. (2020). 24-Month Clinical Evaluation of Different Bulk-Fill Restorative Resins in Class II Restorations. Oper Dent, 45, 123-133. https://doi.org/10.2341/18-144-C. 
Hamano, N., Ino, S., Fukuyama, T., Hickel, R., \& Kunzelmann, K.H. (2013). Repair of silorane-based composites: microtensile bond strength of siloranebased composites repaired with methacrylate-based composites. Dent Mater J, 32(5), 695-701.

Heck, K., Manhart, J., Hickel, R., \& Diegritz, C. (2018). Clinical evaluation of the bulk fill composite QuiXfil in molar class I and II cavities: 10-year results of a RCT. Dent Mater, 34, e138-e147. https://doi.org/10.1016/j.dental.2018.03.023.

Hirata, R., Kabbach, W., de Andrade, O. S., Bonfante, E. A., Giannini, M., \& Coelho, P. G. (2015). Bulk Fill Composites: An Anatomic Sculpting Technique. $J$ Esthet Restor Dent, 27(6), 335-43. https://doi.org/10.1111/jerd.12159.

Hoshino, I. A. E., Bachega, M. de O., Santos, P. H. dos., Briso, A. L., \& Anchieta, R. B. (2021). Influence of increment thickness on microhardness and bond strength in composite resins. Research, Society and Development, 10(9), e32810917974. https://doi.org/10.33448/rsd-v10i9.17974.

Ilie, N., Bucuta, S., \& Draenert, M. (2013). Bulk-fill resin-based composites: an in vitro assessment of their mechanical performance. Oper Dent, 38(6), 618625 .

Ilie, N., \& Hickel, R. (2011). Investigations on a methacrylate-based flowable composite based on the SDR technology. Dent Mater, $27(4), 348-355$.

Kim EH, Jung KH, Son SA, Hur B, Kwon YH, Park JK. Effect of resin thickness on the microhardness and optical properties of bulk-fill resin composites. (2015). Restor Dent Endod, 40(2), 128-35. https://doi.org/10.5395/rde.2015.40.2.128.

Kleverlaan, C. J., \& Feilzer, A. J. (2005). Polymerization shrinkage and contraction stress of dental resin composites. Dent Mater, $21(12), 1150-1157$.

Kruly, P. C., Giannini, M., Pascotto, R. C., Tokubo, L. M., Suga, U. S. G., Marques, A. C. R., \& Terada, R. S. S. (2018). Meta-analysis of the clinical behavior of posterior direct resin restorations: Low polymerization shrinkage resin in comparison to methacrylate composite resin. Plos One, 13 , e0191942. https://doi.org/10.1371/journal.pone.0191942.

Laegreid, T., Gjerdet, N. R., Johansson, A., \& Johansson, A. K. (2014). Clinical decision making on extensive molar restorations. Oper Dent, 39(6), 231-240.

Leprince, J. G., Palin, W. M., Hadis, M. A., Devaux, J., \& Leloup, G. (2013). Progress in dimethacrylate-based dental composite technology and curing efficiency. Dent Mater, 29, 139-156. https://doi.org/10.1016/j.dental.2012.11.005.

Lima, R. B. W., Troconis, C. C. M., Moreno, M. B. P., Murillo-Gómez, F., \& de Goes, M. F. (2018). Depth of cure of bulk fill resin composites: A systematic review. J Esthet Restor Dent, 30(6), 492-501.

Lins, R. B. E., Aristilde, S., Osório, J. H., Cordeiro, C. M. B., Yanikian, C. R. F., Bicalho, A. A., Stape, T. H. S., Soares, C. J., \& Martins, L. R. M. (2019a). Biomechanical behaviour of bulk-fill resin composites in class II restorations. J Mech Behav Biomed Mater, 98, 255-261. doi: 10.1016/j.jmbbm.2019.06.032.

Lins, R., Vinagre, A., Alberto, N., Domingues, M. F., Messias, A., Martins, L. R., Nogueira, R., \& Ramos, J. C. (2019b). Polymerization Shrinkage Evaluation of Restorative Resin-Based Composites Using Fiber Bragg Grating Sensors. Polymers (Basel), 11(5), 859. https://doi.org/10.3390/polym11050859.

Manhart, J., Chen, H. Y., \& Hickel, R. (2010). Clinical evaluation of the posterior composite Quixfil in class I and II cavities: 4-year follow-up of a randomized controlled trial. J Adhes Dent, 12, 237-43. https://doi.org/10.3290/j.jad.a17551.

Mahmoud, S. H., Ali, A. K., \& Hegazi, H. A. (2014). A three-year prospective randomized study of silorane- and methacrylate-based composite restorative systems in class II restorations. J Adhes Dent, 16(3), 285-292.

Monterubbianesi, R., Orsini, G., Tosi, G., Conti, C., Librando, V., Procaccini, M., \& Putignano, A. (2016). Spectroscopic and Mechanical Properties of a New Generation of Bulk Fill Composites. Front Physiol, 7:652.

Moorthy, A., Hogg, C. H., Dowling, A. H., Grufferty, B. F., Benetti, A. R., \& Fleming, G. J. (2012). Cuspal deflection and microleakage in premolar teeth restored with bulk-fill flowable resin-based composite base materials. J Dent, 40(6), 500-5.

Mosharrafian, S., Shafizadeh, M., \& Sharifi, Z. (2019). Fracture Resistance of a Bulk-Fill and a Conventional Composite and the Combination of Both for Coronal Restoration of Severely Damaged Primary Anterior Teeth. Front Dent, 16(1), 69-77. https://doi.org/10.18502/fid.v16i1.1112.

Rueggeberg, F. A., Giannini, M., Arrais, C. A. G., \& Price, R. B. T. (2017). Light curing in dentistry and clinical implications: a literature review. Braz Oral Res, 28, e61. https://doi.org/10.1590/1807-3107bor-2017.vol31.0061.

Rodrigues, L. D., Costa, I. A., Rabelo, Z. H., Oliveira, L. L., Monteiro, R. M. F., Sá, H. C., Estellita, M. C. A., Lima, K. E. R., Lemos, M. V. S., Fontes, N. M., Silva, R. A. D. A., Isaias, P. H. C., Mendes, T. A. D., \& Sales, E. M. A. (2021). Composite resin innovations: a literatura review. Research, Society and Development, 10(3), 1-14. https://doi.org/10.33448/rsd-v10i3.13099

Santos, D. T., Dias, K. R., \& Santos, M. P. (2016). Dental amalgam and its role in current dentistry. Revista Brasileira de Odontologia, 73(1), 64-68.

Sebold, M., Lins, R. B. E., André, C. B., Martins, L. R. M., \& Giannini, M. (2020). Flowable and Regular Bulk-Fill Composites: A Comprehensive Report on Restorative Treatment. Int J Periodontics Restorative Dent, 40(2), 293-300. https://doi.org/10.11607/prd.3932.

Tarle, Z., Attin, T., Marovic, D., Andermatt, L., Ristic, M., Tauböck, TT. (2015). Influence of irradiation time on subsurface degree of conversion and microhardness of high-viscosity bulk-fill resin composites. Clin Oral Investig, 19(4), 831-840. https://doi: 10.1007/s00784-014-1302-6.

Türkün LS, Aktener BO, Ateş M. Clinical evaluation of different posterior resin composite materials: a 7-year report. (2003) Quintessence Int. Jun;34(6):41826. PMID: 12859086 .

Veloso, S. R. M., Lemos, C. A. A., de Moraes, S. L. D., do Egito Vasconcelos, B. C., Pellizzer, E. P., \& de Melo Monteiro, G. Q. (2019). Clinical performance of bulk-fill and conventional resin composite restorations in posterior teeth: a systematic review and meta-analysis. Clin Oral Investig, $23,221-233$. https://doi.org/10.1007/s00784-018-2429-7. 
Research, Society and Development, v. 10, n. 13, e136101320852, 2021

(CC BY 4.0) | ISSN 2525-3409 | DOI: http://dx.doi.org/10.33448/rsd-v10i13.20852

Vildósola, P., Nakouzi, J., Rodriguez, S., Reyes, A., Reyes, J., \& Conejeros, C. (2019). Six month follow-up of two Bulk-fill composites in non-carious cervical lesions: Double blind randomized clinical trial. J Oral Res, 8, 210-219. https://doi.org/10.17126/joralres.2019.032.

Yazici, A. R., Antonson, S. A., Kutuk, Z. B., \& Ergin, E. (2017). Thirty-Six-Month Clinical Comparison of Bulk Fill and Nanofill Composite Restorations. Oper Dent, 42, 478-485. https://doi.org/10.2341/16-220-C.

Yoshikawa, T., Sano, H., Burrow, M. F., Tagami, J., \& Pashley, D. H. (1999). Effects of dentin depth and cavity configuration on bond strength. J Dent Res, 78, 898-905. https://doi.org/10.1177/00220345990780041001.

Zorzin, J., Maier, E., Harre, S., Fey, T., Belli, R., Lohbauer, U., Petschelt, A., \& Taschner, M. (2015). Bulk-fill resin composites: polymerization properties and extended light curing. Dent. Mater, 31, 293-301. https://doi.org/10.1016/j.dental.2014.12.010. 